\title{
Von der Küste bis zu den Alpen Ein Pflegesymposium im nord- und eines im süddeutschen Raum
}

Das Pflegesymposium des AKTX-Pflege findet bereits zum vierten Mal in Nord- und zum zweiten Mal in Süddeutschland statt. Der AKTX-Pflege möchte den Transplantationspflegekräften in ganz Deutschland eine Plattform bieten, um Neues aus der Transplantationsmedizin zu erfahren und Erfahrungen, Ideen und Konzepte austauschen zu können.

Wir freuen uns, Sie in diesem Jahr zu unserem 4. Norddeutschen Pflegesymposium an die Müritz und zu unserem 2. Süddeutschen Pflegesymposium nach München einladen zu dürfen. Unser Bestreben war es, ein ähnliches Symposium im Norden und im Süden unseres Landes anzubieten.

Die Symposien der vergangenen Jahre haben uns gezeigt, wie groß das Interesse von Pflegenden, Ärzten und Patienten an unserer Veranstaltung ist. Damit wir auch in Zukunft weiter hoch qualifiziert und professionell arbeiten können, ist ein Wissens-, Erfahrungs-, Ideen- und Konzeptaustausch sehr wichtig. Mit unserem Symposium ha-

\section{Termine}

\section{Norddeutsches Pflegesymposium 4.-5. April 2008 \\ Klink (Müritz)}

\section{Süddeutsches Pflegesymposium} 11.-12. April 2008

München

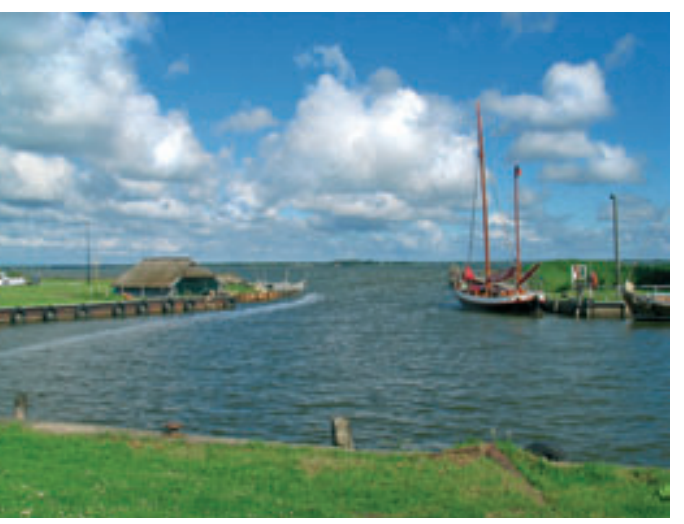

ben wir hierfür eine gute Plattform geschaffen. Auch in diesem Jahr werden wir ein abwechslungsreiches und lehrreiches Programm bei beiden Symposien anbieten.

\section{Von Infektionen und Hauttumoren} Ein nicht zu unterschätzendes Thema im Langzeitverlauf nach Transplantation sind Infektionen. Es gibt lange bekannte Infektionen aber auch neue Erkrankungen auf die wir zunehmend achten müssen. Für dieses sehr interessante Thema konnten wir für die Veranstaltung im Norden Prof. Petra Reinke von der Charité Berlin und Prof. Michael Fischereder von der LMU München für das Symposium im Süden als Referenten gewinnen.

Viel Raum für Diskussionen wird sicherlich das Thema „30 Jahre Transplantation in München: Anfänge, Erfolge, Auswüchse“ von Barbara Gnatz, LMU München, bieten.

Ein Thema, dem man nicht genug Aufmerksamkeit widmen kann, sind Hauttumoren im Langzeitverlauf nach Transplantationen. Dr. Claas Ulrich aus der Charité hat sich sehr intensiv mit diesem Thema beschäftigt und wir werden sicher viele neue Ansätze für unsere tägliche Arbeit mitnehmen können.

Kunsttherapie und Rehabilitation Der Vortrag „Kunsttherapie als besondere Form der psychologischen Betreuung nach Transplantation“ wird uns aufzeigen, wie vielfältig und wichtig die psychologische Betreuung ist. Kornelia Krimmling, Ilka Ahrent und Kerstin Peter von der Müritz Klinik stellen uns diese Form der Therapie vor.

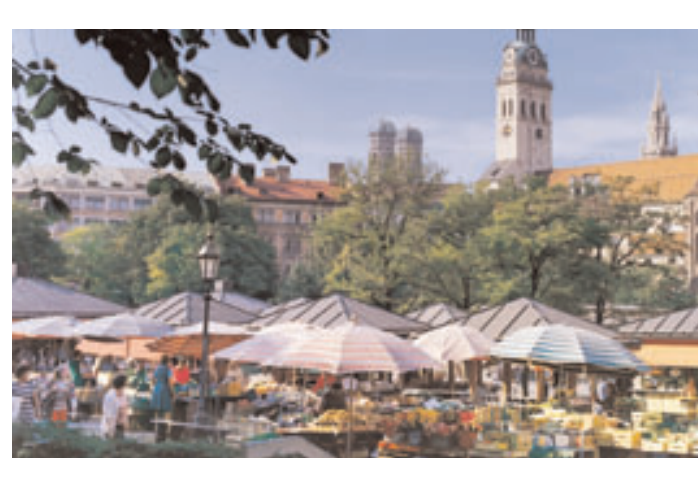

MRSA in der Rehabilitationsklinik - ein Punkt der uns immer mehr beschäftigt. Cornelia Czerwinski wird uns das Konzept der Müritz Klinik vorstellen.

Ein sichtbarer Erfolg nach einer Nierentransplantation ist immer, wenn der erste Urin läuft. Für die Patienten ist es etwas Besonderes, wenn sie sehen, wie sich der Katheterbeutel langsam füllt. Dr. Gottfried May und Petra Hecker von der Berliner Charité werden sich in ihrem Vortrag diesem Thema widmen.

Dr. Dirk Janek aus der Müritz Klinik wird im Süden über die Rehabilitation nach einer Transplantation referieren - ein Thema mit dem wir uns zunehmend in den Kliniken auseinandersetzen müssen. Im Norden wird der Tag mit dem Workshop „Sonografie des Transplantates“ enden, Janek wird den Teilnehmern das Wichtigste hierzu praktisch vorführen. Aufbruch in ein neues Leben, ein Patientenbericht von H. Kohler wird im Süden das Symposium abrunden. Die vollständigen Programme finden Sie unter www.aktxpflege.de oder unter www. rapamune.de.

An dieser Stelle möchten wir der Firma Wyeth sehr herzlich für die Unterstützung danken, ohne deren Hilfe die Symposien nicht in dieser Form denkbar wären. Wir freuen uns auf die Symposien und hoffen, auch Sie begrüßen zu dürfen.

Petra Hecker

1. Vorsitzende

AKTX-Pflege e. V.

Barbara Gnatz Beisitzerin AKTX-Pflege e. V. 